\title{
Belphégor
}

\section{Agnès Giard, Un désir d'humain, les « love doll » au Japon}

\section{Sixtine Audebert}

\section{(2) OpenEdition}

\section{Journals}

Electronic version

URL: http://journals.openedition.org/belphegor/894

DOI: $10.4000 /$ belphegor.894

ISSN: 1499-7185

Publisher

LPCM

Electronic reference

Sixtine Audebert, « Agnès Giard, Un désir d'humain, les « love doll » au Japon », Belphégor [Online],

15-1 | 2017, Online since 09 July 2017, connection on 19 April 2019. URL : http://

journals.openedition.org/belphegor/894 ; DOI : 10.4000/belphegor.894

This text was automatically generated on 19 April 2019

\section{(c) $($ () $\odot$}

Belphégor est mis à disposition selon les termes de la Licence Creative Commons Attribution - Pas d'Utilisation Commerciale - Pas de Modification 4.0 International. 


\title{
Agnès Giard, Un désir d'humain, les « love doll » au Japon
}

\author{
Sixtine Audebert
}

\section{REFERENCES}

Agnès Giard, Un désir d'humain, les «love doll » au Japon, Les Belles Lettres, coll. « Japon », Paris, 2016, 25,90€

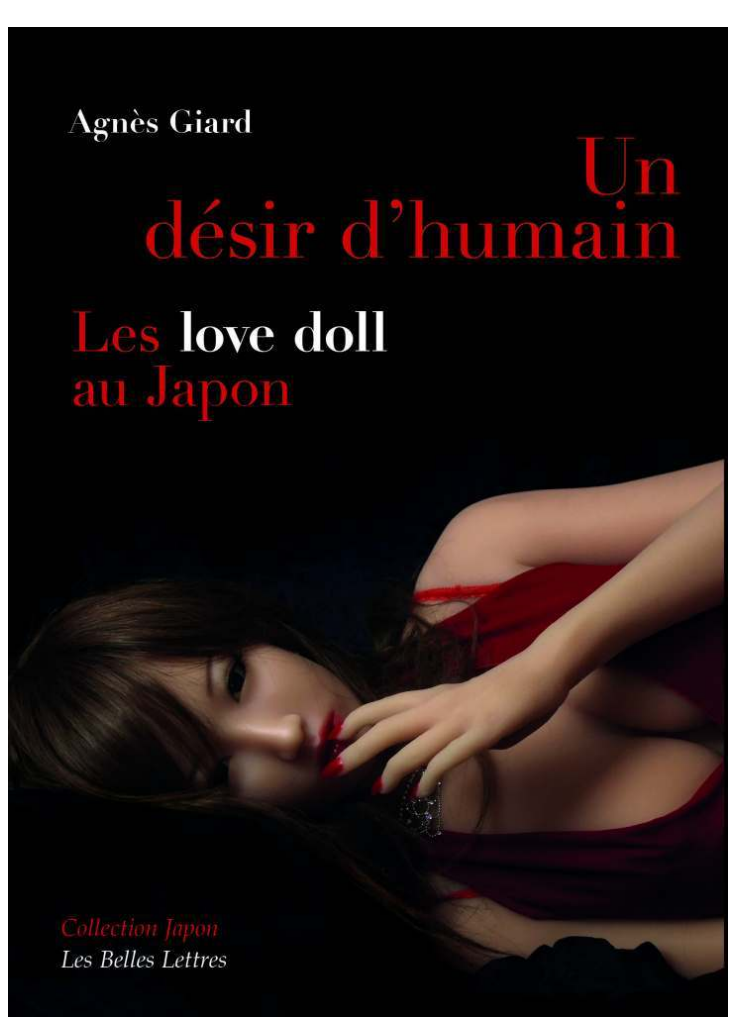


1 Le livre Un désir d'humain, les «love doll » au Japon est le fruit d'une réflexion développée par Agnès Giard dans sa thèse d'anthropologie soutenue en 2015 à Paris X Nanterre, Humanité désirée, humanité simulée: étude de l'effet de présence dans les objets anthropomorphiques au Japon. L'auteure rassemble dans cette somme de nombreux matériaux qui en font un document inédit pour qui s'intéresse aux « poupées d'amour ». Elle s'appuie sur quantité de documents, d'articles de journaux (depuis l'émergence des dolls jusqu'à l'époque la plus contemporaine), ainsi que de nombreux témoignages recueillis auprès des fabricants des différentes firmes nippones et des utilisateurs (elle étudie en particulier les productions narratives et photographiques de ces utilisateurs mises en ligne sur les blogs).

2 La thèse de l'auteure, résumée dans la formule " un désir d'humain », est qu'il est possible d'attribuer des qualités psychologiques humaines à des objets inanimés dans un processus d'investissement affectif. Agnès Giard invite à contourner la distinction entre les domaines matériel et spirituel lorsqu'on parle des «love dolls » au Japon. Cette pratique, le "jeu de la poupée», devient une forme d'animisme raffiné, un exercice spirituel astreignant, à partir duquel le virtuose insufflerait de la vie dans cette chair plastique inerte en faisant de la poupée une compagne. C'est pourquoi Agnès Giard rappelle avant tout que la distinction radicale de la matière et de l'âme est un concept occidental. Dans l'introduction, elle compare ainsi les imaginaires collectifs en exposant «les préjugés concernant la love doll » chez Français et Japonais. Il s'agira par la suite de comprendre l'objet complexe qu'est la poupée en quittant nos réticences d'Européens.

3 Le premier chapitre («Le marketing de la love doll: la love doll comme 'être de substitution' ») est consacré à l'histoire des poupées sexuelles dans la société japonaise et aux discours sur le produit des différents fabricants. il met en évidence la qualité supplétive de la love doll (celle de combler le besoin sexuel, le besoin affectif, ou d'avoir une vertu thérapeutique). La conclusion en est que ce simple objet matériel, pourtant dépourvu d'humanité, a une action psychologique sur l'utilisateur. C'est sans doute ce qui conduit l'auteure à franchir le pas dès le second chapitre et à parler d'« esthétique de la love doll ». Elle s'interroge alors sur les qualités formelles de l'objet (le visage, les yeux) qui en font un support pour la méditation. Ainsi de simple poupée sexuelle, la love doll devient "miroir de l'âme", son anthropomorphisation reposant sur une intellectualisation qui évacue largement la dimension d'une sexualité trop prosaïque. L'objet à l'image de l'homme (ou de la femme plutôt) devient un support permettant à l'individu d'accéder à lui-même.

4 L'auteure est ainsi conduite à parler dans le dernier chapitre de « métaphysique de la love doll », désignant la poupée comme "corps d'emprunt » et dénonçant les limites de l'expérience sensible, incomplète, de l'objet. Elle montre, en s'appuyant principalement sur le vagin extractible, acheté séparément, que c'est une création destinée à autre chose que le sexe, un «véhicule " pour une sorte d'expérience mystique. Dans ce chapitre, l'auteure plante (un peu tardivement) le contexte culturel contemporain. Elle décrit les lois sur la censure dans la pornographie, et montre comment la sous-culture Otaku est liée aux frustrations que fait peser au Japon ladite censure sur les imaginations. Elle fait alors l'apologie d'une sexualité raffinée aux antipodes de la pornographie occidentale, nourrie d'un érotisme délicat de la dissimulation.

5 C'est ainsi que tout en parlant de la love doll, poupée d'apparence féminine produite en série principalement pour le sexe, Agnès Giard en vient à mettre l'accent avant tout sur l'idée d'une projection d'humanité. La position d'Agnès Giard est difficile. En effet, la love 
doll est un objet culturel complexe et digne de l'attention du chercheur. Cependant elle est doublement illégitime : c'est un produit industriel et un objet sexuel. Agnès Giard fait de la poupée le moyen " heuristique » pour comprendre l'hypermodernité japonaise, cet Autre de la culture de consommation occidentale qui synthétise culture de consommation et tradition, religion et artisanat.

Une telle approche ouvre bien des pistes dont on suivra quelques-unes ici. Que certains utilisateurs ne touchent jamais leur poupée (comme le rapporte l'auteure), qu'ils déplacent la pratique sexuelle dans des mises en scène photographiques fantasmatiques, ou que la "vraie chose » (hon-mono, terme japonais pour désigner la pratique sexuelle) ait bien lieu, dans tous les cas, occulter ainsi le sexe et déplacer son intérêt ailleurs, dans un objet «non sexuel » (ou problématiquement sexuel), cela s'appelle du fétichisme. Or, cette paraphilie qui consiste à aimer des représentations anthropomorphiques, statue ou poupée, n'est pas propre à l'Orient. On peut chez nous la faire remonter par exemple à la légende grecque de Pygmalion.

7 Or, tout dans l'histoire culturelle de la poupée sexuelle rapportée par l'auteure et dans le marketing de la love doll la désigne en fait comme un objet à fétichiser. Elle est à la fois un sextoy et tout autre chose. La particularité de la poupée est donc qu'elle est explicitement commercialisée à la fois pour sa valeur d'usage (ce qu'induit le sextoy), mais que l'accent est mis par ses producteurs mêmes sur sa valeur d'échange. La love doll est très chère, très belle, ce qui pour certains l'apparente même à une œuvre d'Art. Elle a plus généralement les caractéristiques de l'artisanat et du luxe, c'est-à-dire de la marchandise fétichisée, ce que confirme le fait que certains la collectionnent. Enfin, elle joue sur les frontières entre réel et imaginaire, pouvant satisfaire à la fois le besoin de sexe et de présence humaine. Elle devient « être de substitution », ou encore fétiche dans la définition anthropologique, statuette « religieuse » faisant le lien avec un inaccessible transcendant.

8 Pour comprendre un tel rapport à ce bien de consommation très particulier, il faut rappeler les mutations des pratiques culturelles de la société postmoderne. Celles-ci sont caractérisées par la mise en crise des grands récits de l'amour et du sexe (comme le mariage) au profit de micro récits produits par l'individu (le consommateur masculin, et non l'institution et la famille comme dans la société moderne). Lorsqu'il réalise une mise en scène photographique, il manipule des signes (associé à des scénarios érotiques, ou romantiques, ou quotidiens à partir d'objet/poses/titres les signifiant). Les idylles avec ces poupées aux têtes interchangeables dont on explicite le caractère factice sont ces scenarii, sources virtuelles d'une infinité de micro récits. Le caractère fictionnel et ludique est à son tour souvent explicité par la mise en scène de la poupée sur un mode parodique sur les blogs des dollers ${ }^{1}$. Ainsi la photographie d'une poupée brandissant un fer à repasser reconstitue-t-elle le stéréotype de la femme au foyer; celle d'une poupée couverte de mousse masquant son sexe reprend le topos de la jeune fille surprise nue au bain (en la présentant, dans le même temps, souillée par le regard de l'homme)... Ce sont les images partagées d'un imaginaire érotique masculin qui détermine le caractère vraisemblable de la poupée et unifie son corps composite en un idéal de femme. En effet, ce n'est pas parce que ces poupées servent de supports donnant une cohérence au fantasme grâce à la qualité de leur ressemblance qu'elles ne sont plus des objets - autant dire, qu'elles sont des femmes ${ }^{2}$.

9 L'esthétique formelle idéale évoquée dans la deuxième partie peut être rapprochée de celle de la marchandise postfordiste. Ce corps en pièces détachées est un modèle sérialisé soumis aux lois du marché : en raison du coût du produit, seuls sont gardés les modèles à 
succès, excepté dans le cas de commandes singulières. Mais c'est aussi, suivant la logique postfordiste, un modèle ultra personnalisable, d'abord lors de l'acte d'achat (offrant des options classiques pour les yeux, les cheveux, mais aussi des choix plus minutieux pour les poils pubiens, la couleur des tétons, etc....) ; puis, à la maison, dans l'usage de la poupée (il est possible de changer de perruque, de tenue...). On voit bien comment tous les détails prennent une grande importance et sont « fétichisés »: ils deviennent tous signifiants, puisque le consommateur aime et sélectionne chacun d'entre eux comme des traits déterminant son désir, comme ce qui le fait fantasmer. Cela correspond aux «éléments d'attraction " décrits par Hiroki Azuma ${ }^{3}$. Le retour de ces éléments d'attraction et leur reconnaissance par le consommateur entrent en jeu dans la création d'affects et l'élaboration émotionnelle à partir de la poupée. Par la suite, libre au propriétaire de la love doll de composer à partir de sa «base de données » (Azuma) $)^{4}$ non pas un corps, mais des corps, non pas une poupée, mais des poupées; et à partir de ces supports de fictionnalisation, une multiplicité de récits exploitant ces éléments fétichisés.

C'est pourquoi il est important d'avoir lié, comme le fait l'auteure dans la troisième partie du livre, la love doll à d'autres productions de la culture japonaise contemporaine, sexualisées de manière plus ou moins explicite : les hérö̈nes de manga (toujours associées au type kawaii), les mascottes, les idoles, les eroge (erotic games, et autres jeux de lecture et de séduction)... Agnès Giard prend soin d'associer dans le même temps la fascination des poupées à la sous-culture de ces «obsessionnels » que sont les otaku, selon les mots de l'auteure. En suivant les analyses développées dans ce chapitre concernant les lois sur la censure s'appliquant à ces productions érotiques, on peut parler de fétichisme, selon l'acception psychanalytique du mot cette fois : à cause de l'interdiction de représentation des organes génitaux, le sexuel est déplacé et investi dans tout l'environnement. Ainsi une nouvelle forme d'affirmation de la virilité passant essentiellement par la consommation culturelle de produits «mettant en jeu de manière oblique la sexualité produit-t-elle paradoxalement une esthétique de la femme toujours vierge, la petite fille intouchable, à travers cette fantasmatique "pédocriminelle» du viol dont il est rapidement question dans les dernières pages de l'ouvrage.

\section{NOTES}

1. Terme communautaire par lequel se désigne les utilisateurs de poupée.

2. Ou alors quelle conception de la femme propose-t-on? Nous ne pouvons ici nous empêcher de citer un des producteurs de love dolls dont Agnès Giard rapporte les propos dans la sous-partie consacrée à la question de l'échec commercial de poupées d'apparence masculine : « la poupée ne peut être qu'une femme parce que seuls les hommes (hétérosexuels) ont besoin d'amour. Les femmes (et les gays), eux, ont simplement besoin de plaisir sexuel. » (sic).

3. Azuma, Hiroki, Génération Otaku, les enfants de la postmodernité, Hachette, « Haute tension ", 2008

4. Ibid. La "base de données" serait ici constituée par les différentes perruques, tenues et accessoires, ces « éléments d'attraction » soigneusement acquis par l'utilisateur. 